\title{
Diseño y construcción de una secadora automática para cacao a base de aire caliente tipo rotatorio para una capacidad de $500 \mathbf{~ k g}$
}

\section{(Design and construction of an automatic dryer for cacao a rotating type of hot air base for a capacity of $500 \mathrm{~kg}$ )}

\author{
Javier Orna ${ }^{1}$, Nelson Chuquín ${ }^{1}$, Luis Saquinga ${ }^{1}$, Oswaldo Cueva ${ }^{1}$
}

\begin{abstract}
Resumen:
El incremento de tiempo en el proceso de secado de cacao y la problemática temporada invernal motivaron el desarrollo de una máquina secadora automática para cacao que cumpla con las características de secado, reduciendo la humedad del producto a un porcentaje adecuado en la Comunidad Mirador Lojano ubicada en la provincia de Santo Domingo de los Tsáchilas. El desarrollo del proyecto tiene como alcance diseñar y construir una secadora automática para cacao a base de aire caliente tipo rotatorio para una capacidad de $500 \mathrm{~kg}$; esto implicó la recopilación de información necesaria para realizar la metodología de diseño, el estudio de alternativas tecnológicas existentes en el mercado y seleccionar la más adecuada que cumpla con los requerimientos del usuario, parámetros de calidad y producción. Posteriormente se realizó el diseño mecánico y energético de los elementos que conforman la máquina. En conclusión, la máquina secadora automática para cacao logra un secado adecuado del $9 \%$ de humedad en un período de 4 a 5 horas durante el proceso, mejorando así la eficiencia y producción.
\end{abstract}

Palabras clave: eficiencia energética; diseño mecánico; secado; cacao.

\begin{abstract}
:
The increase of time in the process of dry of cocoa and the problematic winter season motivated in realizing an automatic drying machine for cocoa that fulfills the characteristics of dry, reducing to a percentage of adequate humidity in the Mirador Lojano Community located in the Province of Santo Domingo of the Tsáchilas. The development of the project has as its scope to design and build an automatic dryer for cocoa based on hot air rotary type for a capacity of $500 \mathrm{Kg}$; this involved the collection of information necessary to carry out the design methodology, the study of existing technological alternatives in the market and select the most viable one that meets the user's requirements, quality and production parameters. Subsequently the mechanical and energetic design of the elements that make up the machine was made. In conclusion, the automatic drying machine for cocoa achieves an adequate drying of $9 \%$ humidity in a period of 4 to 5 hours during the process, thus improving the efficiency and production.
\end{abstract}

Keywords: energy efficiency; mechanical design; drying; cacao.

\section{Introducción}

El cacao es un producto muy importante de exportación en el Ecuador, su cultivo se lo realiza durante el año completo, por ser un producto de extremada producción la mayoría de personas del sector costero se dedican al cultivo de cacao.

Después de la cosecha es importante realizar lo que se refiere a la fermentación, aquí el cacao posee un porcentaje de humedad que varía entre 55 y $60 \%$ aproximadamente, hay diferentes maneras de hacerlo sean estos mediante cajones de

\footnotetext{
${ }^{1}$ Escuela Superior Politécnica de Chimborazo, Riobamba, Ecuador ( \{javier.orna, nelson.chuquin, luis.saquinga, oswaldo.cueva\} @espoch.edu.ec .
} 
madera o tendales (JÁCOME BAZURTO, 2010). Otro parámetro a considerar con el propósito de reducir el porcentaje de humedad del cacao es mediante el secado, para que su resultado final tenga éxito en el mercado es lograr un porcentaje de humedad entre 9 y $10 \%$ aproximado, a esta humedad el cacao mantiene sus características, la temperatura debe alcanzar un rango de $40-60{ }^{\circ} \mathrm{C}$ para el secado adecuado. (ESPINOZA MARTÍNEZ \& RUIZ BUITRÓN, 2015)

Para llevar a cabo el secado se lo realiza ya sea de forma natural o artificial, cuando se lo realiza por secado natural se aprovecha de la radiación solar, pero en época invernal es perjudicial ya que ocasiona daños en el producto y pérdidas en la producción. El tiempo de secado de forma natural lleva de 6 a 7 días según la radiación solar (600 a $800 \mathrm{~W} / \mathrm{m} 2$ ), temperatura, humedad relativa, etc. (García A, y otros, 2017) La manera de realizar el secado en menor tiempo es de forma artificial ya sea mediante el uso de máquinas artesanales.

Al evidenciar ciertas falencias en la Comunidad Mirador Lojano ubicada en la provincia de Santo Domingo de los Tsáchilas se lo realizará el diseño de una secadora automática para cacao con el objetivo de disminuir el tiempo de secado y aumentar la producción, así como también mejorar la eficiencia energética.

\subsection{El cacao y sus características}

El cacao fino de aroma presenta características adecuadas para la fabricación de chocolate, a nivel mundial representan un $5 \%$ en la producción, es uno de los de mayor exportación ecuatoriana ya que el $75 \%$ es de cacao fino de aroma mientras que el $25 \%$ se refiere al cacao CCN-51. (JIMBO YÉPEZ \& ARBOLEDA MUÑOZ, 2016)

Para el respectivo estudio se van analizar los dos tipos de cacao que son los siguientes: Cacao fino de aroma y cacao CCN-51(Colección Castro Naranjal). (JÁCOME BAZURTO, 2010), en la Tabla 1 se muestra las características del cacao fino de aroma.

Tabla 1. Características del cacao fino de aroma

\begin{tabular}{|l|l|l|}
\hline Características & Fermentada & Sin fermentar \\
\hline Aroma & Agradable & Desagradable \\
\hline Sabor & Medianamente amargo & Astringente \\
\hline Forma & Hinchada & Aplanada \\
\hline Color interno & Café oscuro & Café violáceo \\
\hline Textura & Quebradiza & Compacta \\
\hline $\begin{array}{l}\text { Separación de la } \\
\text { testa }\end{array}$ & Fácil & Difícil \\
\hline
\end{tabular}

\subsection{Procesos y tecnologías de secado}

Existen dos procesos de secado para cacao de los cuales son: secado natural y secado artificial.

Secado natural. Este tipo de secado se lo realiza por medio de la radiación solar ya sea mediante el uso de tendales (madera/cemento), en donde los rayos del sol inciden directamente sobre la superficie, el producto se encuentra en montículos. (JORDÁN RUBIO, 2013)

Este tipo de secado conlleva mayor tiempo y área extensa para realizarlo, el cual en las épocas de lluvia el proceso se detiene, y el producto puede deteriorarse ocasionando pérdidas en la producción.

Secado artificial. Para la aplicación de este tipo de secado se requiere el uso de máquinas en donde se optimiza el tiempo de secado y la mano de obra, sobre todo en donde no es posible realizar el secado natural debido a la baja incidencia del sol para dicho proceso específicamente en temporadas invernales donde existen variaciones climáticas. Se debe tener en cuenta que con este método el área de secado es menor a 
la que se utiliza con el secado natural de esta manera consiguiendo incremento en la producción. (GONZALÉS VÁZQUES, 2012)

Entre las posibles máquinas existentes en el Ecuador y a nivel mundial más utilizadas para el secado de cacao en la actualidad son las siguientes:

Secadora de cacao rectangular. Este tipo de máquina es muy utilizado por los productores de cacao debido a su tiempo de secado y ahorro de energía, la remoción del grano se lo realiza de forma manual, sea por medio de palas de madera o metal, el diseño de la secadora dan un buen resultado de productividad, la máquina consta de una cámara de secado en la parte inferior, el material para su ensamble puede ser de acero inoxidable o galvanizado por tratarse de un producto alimenticio, hay un control de llama para regular la temperatura, el combustible para el equipo que se suele utilizar es el GLP o el diésel, además consta de compuertas para la descarga y limpieza del producto.

Secadora de cacao cilíndrica vertical. Este tipo de máquina permite obtener el secado de cacao en el menor tiempo posible y se diferencia de las rectangulares en la manipulación del grano con la utilización de elementos y mecanismos en una forma mecánica. Su diseño para el proceso de secado da como resultado un equipo de elevada productividad sin variar su calidad. La secadora cuenta con: plataforma propia para aprovechamiento del calor, diseño ensamblado con materiales de acero inoxidable alimenticio o galvanizado, puede ser opcional; su temperatura se regula electrónicamente, consta también con puertas de descarga para el vaciado del producto.

Sistemas para el secado de cacao. Los sistemas que se van a considerar para realizar el proceso están basados en el calentamiento y ventilación.

\subsection{Ciclo de secado para el cacao}

Si se considera que la materia prima se seca por convección (calor suministrado únicamente por la corriente de aire), los productos aumentan su humedad relativa cuando se enfría con el aire caliente sin llegar a su saturación, ya que puede seguir absorbiendo humedad. En la Figura 1 se muestra el ciclo de secado.

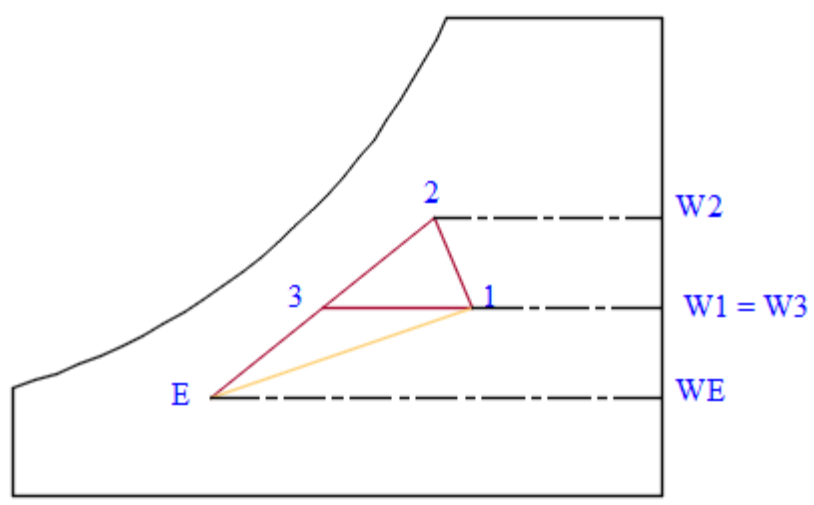

Figura 1. Esquema psicrométrico del proceso de secado

\subsection{Sistemas para el secado de cacao}

Los sistemas que se van a considerar para realizar el proceso están basados en el calentamiento y ventilación.

Calentamiento. Para realizar el proceso de calentamiento se consigue mediante el uso de combustibles, para ello es necesario entender la combustión.

Combustión. Es un proceso de reacción química que consiste en oxidar un elemento, en la combustión intervienen tres factores importantes como es la energía de activación, combustible y comburente. Para su composición el comburente se utiliza el 21 $\%$ de Oxígeno y el $79 \%$ de Nitrógeno, los combustibles pueden ser sólidos, líquidos o 
gaseosos según el uso adecuado para su proceso. (PLAZA ESPINOZA \& YANGUE ZAMBRANO, 2012)

Ventilación. Para la circulación de aire dentro de la cámara de secado se lo realiza de dos maneras ya sea por circulación natural o forzada.

Circulación natural. Este tipo de circulación se da por la diferencia de temperaturas, se emplea en sistemas pequeños que no requieren de mucho aire para su proceso, el rango de la velocidad del aire varía entre $0,4-1 \mathrm{~m} / \mathrm{s}$.

Circulación forzada. La circulación forzada se emplea para grandes capacidades de secado el cual es generado mediante un ventilador, además se puede manipular la velocidad en el proceso de secado, el rango a emplearse va de $0,5-1 \mathrm{~m} / \mathrm{s}$.

\subsection{Materiales más usados para la fabricación de máquinas secadoras de cacao}

Los materiales más usados que se emplean para la construcción de máquinas que intervienen directamente con alimentos a nivel mundial y local son los aceros inoxidables y los galvanizados.

Aceros inoxidables. Los aceros inoxidables por su excelente resistencia a la corrosión se utilizan en la industria alimenticia, tienen una alta gama de disponibilidad. El acero también dependerá de las tensiones que está sujeto, así como formabilidad, maquinabilidad, dureza, soldabilidad y coste. (HAUSER, y otros, 2004)

Acero inoxidable AISI 304. Este acero es el más utilizado por las propiedades de soldadura y conformado que presenta; el más versátil es de la serie 300 , además de tener excelente resistencia a la corrosión. (TIRIRA SUÁREZ \& CASTRO MACAS, 2014)

\section{Metodología}

Para el diseño y construcción de la secadora automática para cacao se basó mediante fases e interacciones del proceso de diseño. Los pasos para realizar el proceso son los siguientes:

Identificación de la necesidad. En la primera fase se refiere la necesidad del usuario, para ello el enunciado será breve y sin detalle. Un ejemplo en este caso sería realizar la secadora de cacao debido al incremento de tiempo que lleva en el proceso de forma natural, para lo cual se considerará la velocidad de aire, temperatura y humedad para el proceso y así obtener un producto de calidad. (BUDYNAS \& NISBETT, 2014)

Investigación preliminar. Esta fase es una de las más importantes en el desarrollo del proceso, es aquí donde se realiza una investigación profunda, se analiza si el problema a plantearse aún no ha sido resuelto, si la solución que se requiere se lo encuentra en el mercado, si es más económico adquirirlo que crear una solución propia. Es necesario realizar una investigación del arte la cual está asociado con productos parecidos y tecnologías, o en muchos casos ciertas empresas aplican el benchmarking (compara y mejora el producto). (NORTON, 2009)

Diseño conceptual. Es la parte fundamental del diseño donde se plantean las posibles soluciones para conseguir el diseño final del producto.

Diseño preliminar. Esta etapa es de determinación y concretización de las componentes en su forma específica, materiales propuestos, el tiempo de funcionamiento, las velocidades de los diferentes sistemas, las dimensiones de los planos de conjuntos de dicho diseño, ya sea simples o complejas. (BUDYNAS \& NISBETT, 2014)

En el dimensionamiento de los planos de conjunto de la máquina, se aplican las diferentes ecuaciones, extraídas de los diferentes libros de diseño de elementos de máquina y mecanismos. Sus respectivos análisis y comprobaciones se aplican mediante software CAD. 
Diseño detalle. Este comprende el ensamble total del dibujo detalladamente, documentos de diseño realizados por computadora, cada uno de los elementos especificados con las dimensiones y material. (NORTON, 2009)

El diseño de detalle es la última etapa de proceso que tiene por objeto el despliegue de todos los documentos necesarios para la fabricación del producto. (RIBA ROMEVA, 2002)

Documentación. Esta etapa se recopila toda la información procesada, de forma ordenada, para la presentación final. Aplicación de la norma INEN (Documentación, presentación de tesis, trabajos de grado y otros trabajos de investigación).

Solución. Es una etapa final al problema planteado, pero en muchos de los casos no hay una solución, por el contrario hay varias soluciones que serán correctas y otras que no funcionarán en la ingeniería de diseño es decir no hay una respuesta correcta razón por la cual lo hace interesante. (NORTON, 2009)

Protocolo de pruebas. Establecen los lineamientos a seguir en las pruebas realizadas para verificar el cumplimiento de los requerimientos establecidos por el cliente final.

Explica cómo se hizo la investigación. Se debe describir en forma precisa el procedimiento realizado para comprobar la hipótesis y los recursos empleados en ello. Una investigación debe poder validarse, y en esta sección se proporciona la información necesaria para que el experimento pueda repetirse.

\subsection{Modelado geométrico}

Mediante el uso de herramientas CAD (Solidworks) se realizó el modelado geométrico de las piezas que conforma la máquina como se observa en la Figura 2.

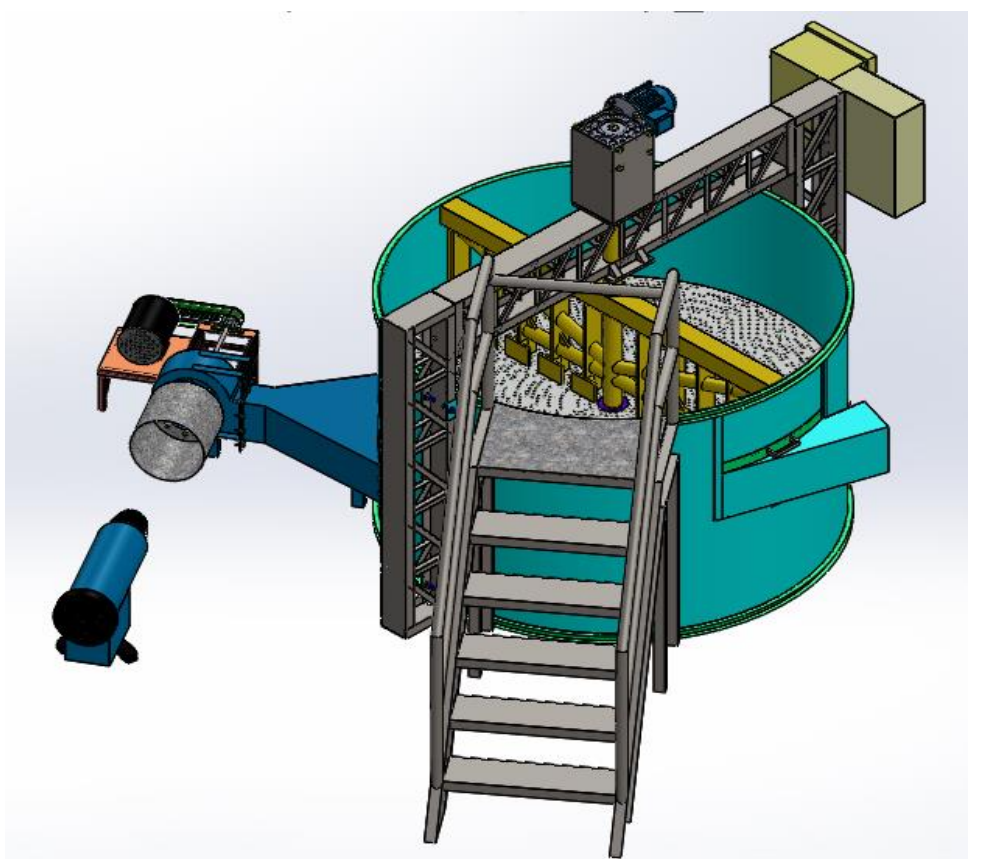

Figura 2. Modelado de la máquina secadora automática para cacao

En la Tabla 2 se detallan los elementos que componen la máquina con la cual se realizará el secado del grano de cacao.

La máquina se construirá con una capacidad de $500 \mathrm{Kg}$, la cual tiene una altura de $210 \mathrm{~mm}$ que garantiza el secado del grano. En el interior el flujo de aire caliente se realiza mediante un quemador y un ventilador centrífugo, con una velocidad de $1,5 \mathrm{~m} / \mathrm{s}$. (ESPINOZA MARTÍNEZ \& RUIZ BUITRÓN, 2015). 
Por mayor comodidad, precio y rendimiento se selecciona el combustible GLP, el cual está compuesto de propano y butano. Este tipo de combustible es más eficiente ya que la utilización del mismo no afecta las propiedades de aroma del cacao durante el secado, este sistema mejora la productividad ya que es menos costoso en comparación a los intercambiadores de calor o mediante resistencias eléctricas.

Además, para el funcionamiento de la máquina se instala un circuito de potencia y control las cuales serán encargadas del encendido del variador de frecuencia, motorreductor, blower, quemador y control adecuado de la temperatura en el interior del contenedor.

Tabla 2. Esquema de Secadero de Cacao y componentes

\begin{tabular}{|l|l|l|}
\hline ESQUEMA DE LA MÁQUINA & COMPONENTES \\
\hline & $\begin{array}{l}\text { 1. Contenedor } \\
\text { 2. Estructura de soporte } \\
\text { base } \\
\text { 3. Estructura pórtico } \\
\text { 4. Estructura escalera } \\
\text { 5. Eje } \\
\text { 6.Sistema removedor } \\
\text { 7. Ventilador } \\
\text { 8. Quemador } \\
\text { 9. Compuerta de vaciado } \\
\text { 10. Motorreductor } \\
\text { 11. Variador de frecuencia } \\
\text { 12. Tamiz } \\
\text { 13. Motor para ventilador } \\
\text { 14. Entrada de aire } \\
\text { 15. Tablero de control }\end{array}$ \\
\hline
\end{tabular}

\subsection{Proceso de diseño} máquina.

Para el proceso de secado se realiza el diseño mecánico y energético de la

\subsubsection{Diseño mecánico}

Con la finalidad de realizar un secado uniforme en el cacao para el presente análisis de determinó como elementos críticos el sistema removedor, el cual se acopla al motorreductor y a la base estructural así como a la estructura del pórtico, en este elemento es donde se aplica mayor carga al ser removidos durante el proceso.

La carga aplicada en el sistema removedor es de $7350 \mathrm{~N}$, valor obtenido por métodos experimentales, por efecto del cacao en el interior del cilindro a ser removido. En la Figura 3 se muestra un esquema del sistema.

Para la fuerza aplicada en las paletas se tiene:

$$
\frac{F_{1}}{920}=\frac{F_{2}}{740}=\frac{F_{3}}{560}=\frac{F_{4}}{380}=\frac{F_{5}}{200}
$$

Se ha considerado la mitad de la carga respecto a la simetría del eje. Se ha realizado un proceso iterativo mediante el Excel, hasta tener un aproximado a la carga deseada, el cual se asume un valor de carga a una de las fuerzas en este caso es a F2.

Donde:

$$
\begin{gathered}
F_{1}=1207,5 \mathrm{~N} \\
F_{2}=971,25 \mathrm{~N} \\
F_{3}=735 \mathrm{~N} \\
F_{4}=498,75 \mathrm{~N} \\
F_{5}=262,5 \mathrm{~N}
\end{gathered}
$$




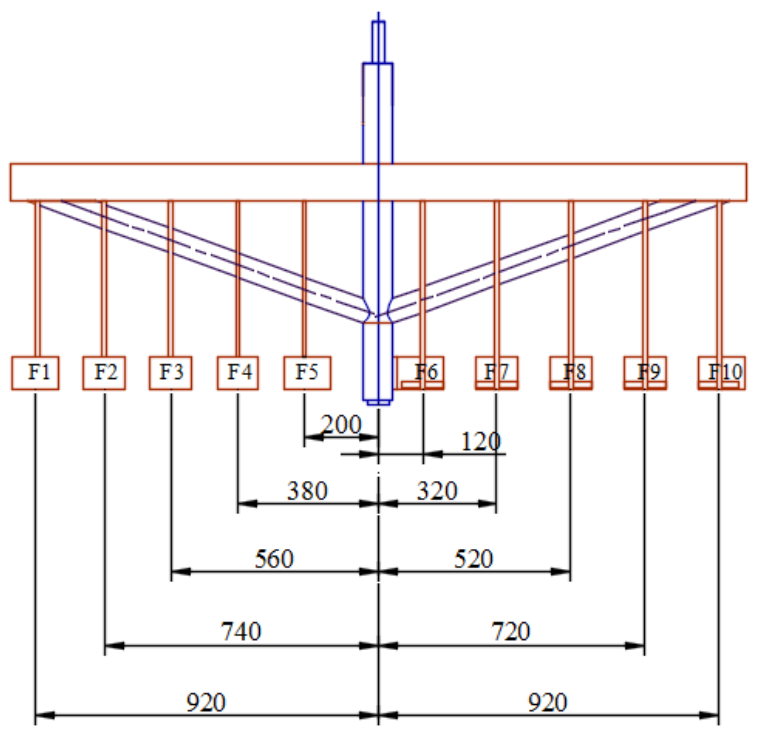

Figura 3. Diagrama del sistema removedor

Con las fuerzas encontradas se aplica de igual forma al lado de la simetría respecto al eje para tener uniformidad en el proceso de remoción del grano. Lo que daría igualdad de fuerzas. Para el análisis de resistencia del material al sistema se selecciona un acero inoxidable AISI 304, una vez determinadas las fuerzas que van ser aplicadas en los removedores, mediante el software ANSYS módulo Static Structural, se consigue un diseño adecuado. En el análisis se obtiene un esfuerzo de Von mises 170,41 MPa, la deformación de 6,4253 mm y un factor de seguridad de 1,8191

\section{Cálculo potencia}

Para la selección del motorreductor se requiere la potencia que necesita el sistema motriz, se debe tomar en cuenta la fuerza aplicada en los removedores, además de los momentos de inercia de cada uno de los elementos que están en rotación acoplados al sistema. El momento de inercia del sistema removedor con respecto al eje y mediante software SolidWorks es de $39,32 \mathrm{Kgm}^{2}$

El momento del sistema removedor se determina mediante:

$$
\begin{gathered}
M_{s r}=I_{s r}{ }^{*} \alpha \\
M_{s r}=5,11 \mathrm{Nm}
\end{gathered}
$$

En la Tabla 3 se muestran los momentos aplicados al sistema removedor.

Tabla 3. Momentos aplicados en los removedores

\begin{tabular}{|l|r|r|r|}
\hline Designación & Fuerza (N) & Distancia (m) & Momento (Nm) \\
\hline M1 & 1207,50 & 0,92 & 1110,90 \\
\hline M2 & 971,25 & 0,74 & 718,73 \\
\hline M3 & 735,00 & 0,56 & 411,60 \\
\hline M4 & 498,75 & 0,38 & 189,53 \\
\hline M5 & 262,50 & 0,20 & 52,50 \\
\hline M6 & 262,50 & 0,12 & 31,50 \\
\hline M7 & 498,75 & 0,32 & 159,60 \\
\hline M8 & 735,00 & 0,52 & 382,20 \\
\hline M9 & 971,25 & 0,72 & 699,30 \\
\hline M10 & 1207,50 & 0,92 & 1110,90 \\
\hline \multicolumn{3}{|c}{} \\
\hline
\end{tabular}


El momento aplicado en los removedores es de:

$$
M_{\text {remov }}=4866,76 \mathrm{Nm}
$$

Momento de todo el sistema de rotación:

$$
\begin{gathered}
M_{\text {sistema }}=M_{s r}+M_{\text {remov }} \\
M_{\text {sistema }}=4871,87 \mathrm{Nm}
\end{gathered}
$$

Velocidad angular de trabajo:

Las máquinas secadoras de cacao trabajan con velocidades bajas, se recomienda entre $(3-6) \mathrm{rpm}$, ya que el movimiento del grano debe ser lento para rapidez de secado, en este caso se trabajará con 3 rpm.

$$
W=3 R P M=0,314 \frac{\mathrm{rad}}{\mathrm{s}}
$$

Potencia de trabajo:

$$
\begin{gathered}
P=M_{\text {sistema }}{ }^{*} W[\mathrm{HP}] \\
P_{T}=2,05 \mathrm{HP}
\end{gathered}
$$

Selección del motorreductor:

A la potencia de trabajo se multiplica por un factor de servicio, el cual depende de las condiciones y características de uso.

Donde:

$F S=1,25$ con choque moderado hasta 10 horas

Con este dato se determina la potencia requerida:

$$
\begin{gathered}
P_{\text {REQUERIDA }}=P_{T}{ }^{*} f_{S} \\
P_{\text {REQUERIDA }}=2,56 \mathrm{HP} \approx 3 \mathrm{HP}
\end{gathered}
$$

\section{Diseño del eje}

En la Figura 4 se muestra el diagrama de cuerpo libre del eje del sistema removedor.

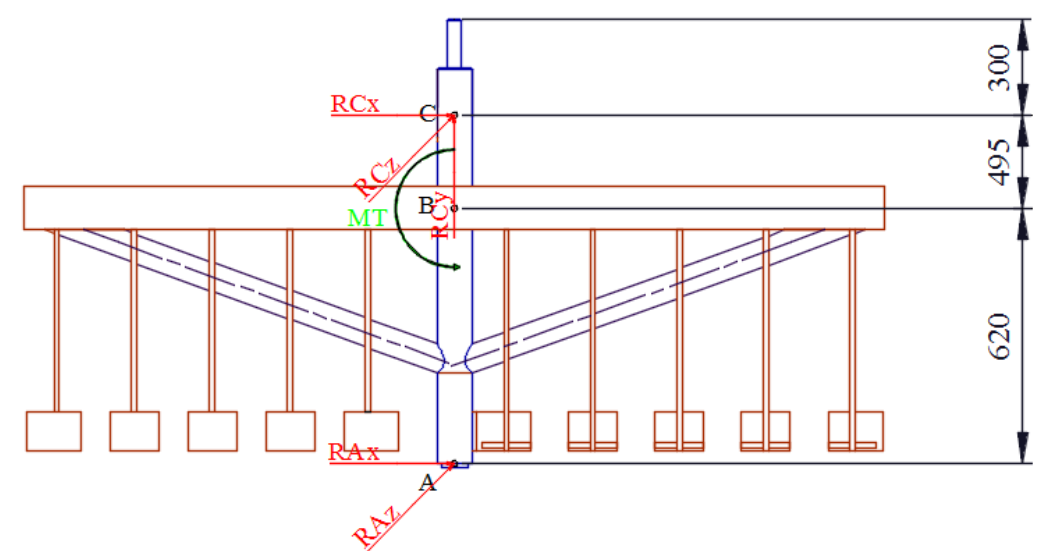

Figura 4. Diagrama de cuerpo libre del sistema

Para determinar el momento torsor se calcula con la potencia requerida, donde se conoce la potencia de 3HP y la velocidad angular de 3rpm.

Donde:

$$
P_{R}=M_{T}{ }^{*} W
$$


Despejando se tiene:

$$
M_{T}=7127,39 \mathrm{Nm}
$$
fuerzas.

Existe una carga axial en el sistema el cual va ser cero al aplicar la sumatoria de

Diseño estático del eje:

EI material utilizado para la construcción del eje es acero inoxidable AISI 304, que tiene como límite de fluencia el valor de $S_{y}=310 \mathrm{MPa}$

Para el criterio de Von - Mises se obtiene un esfuerzo equivalente:

$$
\sqrt{\sigma_{x}^{2}+3 \tau_{x y}^{2}}=\sigma_{e q}
$$

Al remplazar en la ecuación de Von-Mises para $\mathrm{n}=3$ se tiene:

$$
\begin{gathered}
\sqrt{\left(\frac{57090,33}{d^{3}}\right)^{2}+3\left(\frac{36299,5}{d^{3}}\right)^{2}}=\frac{310 \times 10^{6}}{3} \\
d=0,09 m=90 \mathrm{~mm}=3,44 \mathrm{in} \\
\mathrm{d}=3 \mathrm{in}
\end{gathered}
$$

El diámetro obtenido es de 3,44 pero en el mercado se consigue de 3 in, el cual se utilizará para el diseño.

Diseño a fatiga:

Mediante el esfuerzo equivalente de Von Mises

$$
\sigma_{e q}=\sqrt{\left(K f_{f} \sigma_{x}\right)^{2}+3\left(K f_{\tau} \tau_{x y}\right)^{2}}
$$
Soderberg

Cuando el esfuerzo medio es distinto de cero se puede utiizar la ecuación de

$$
\frac{\sigma_{e q a}}{S e}+\frac{\sigma_{e q m}}{S y}=\frac{1}{\eta}
$$

Realizando los cálculos necesarios se obtiene finalmente un coeficiente de seguridad de $\eta=1.2$

\section{Diseño energético}

Para realizar el secado se necesita del calor necesario dentro del cilindro. (ZABALA, 2012)

Las propiedades del aire se determinan mediante el uso del software (EES), en la Tabla 4 y la Tabla 5 se muestran las propiedades al inicio y final del proceso de secado.

Flujo de energía para calentar el aire:

$$
\begin{gathered}
\dot{Q}_{\text {aire }}=\dot{m}_{\text {aire }}\left(h_{2}-h_{1}\right) \\
\dot{Q}_{\text {aire }}=5,37 \frac{K g}{\mathrm{~s}}(106,1-69,75) \frac{\mathrm{KJ}}{\mathrm{Kg}} \\
\dot{Q}_{\text {aire }}=195,19 \mathrm{KW}
\end{gathered}
$$

Con el diseño mecánico y energético se establecen las características generales de la máquina, en la Tabla 6, se muestran las características generales de la máquina. 
Tabla 4. Propiedades del aire al inicio del secado

\begin{tabular}{|l|l|l|}
\hline PROPIEDAD & VALOR & UNIDAD \\
\hline Presión atmosférica (Patm) & 92,64 & $\mathrm{KPa}$ \\
\hline Temperatura de bulbo seco (Tbs) & 25 & ${ }^{\circ} \mathrm{C}$ \\
\hline Humedad relativa $(\mathrm{Hr})$ & 80 & $\%$ \\
\hline Humedad absoluta $(\mathrm{W})$ & 0,0175 & \\
\hline Volumen específico $(\mathrm{v})$ & 0,9498 & $\mathrm{~m}^{3} / \mathrm{Kg}$ \\
\hline Entalpía & 69,75 & $\mathrm{KJ} / \mathrm{Kg}$ \\
\hline
\end{tabular}

Tabla 5. Propiedades del aire al final del secado

\begin{tabular}{|l|l|l|}
\hline PROPIEDAD & VALOR & UNIDAD \\
\hline Presión de secado (Psec) & 92,64 & $\mathrm{KPa}$ \\
\hline Temperatura de secado (Tsec) & 60 & ${ }^{\circ} \mathrm{C}$ \\
\hline Humedad relativa (Hr) & 12,71 & $\%$ \\
\hline Humedad absoluta (W) & 0,0175 & \\
\hline Volumen específico (v) & 1,061 & $\mathrm{~m}^{3} / \mathrm{Kg}$ \\
\hline Entalpía & 106,1 & $\mathrm{KJ} / \mathrm{Kg}$ \\
\hline
\end{tabular}

Tabla 6. Características generales

\begin{tabular}{|l|}
\hline Máquina secadora automática para cacao. Características generales \\
\hline Dimensiones generales: \\
Alto (caja de control) $=1,10 \mathrm{~m}$ \\
Alto para la alimentación de cacao $=1,22 \mathrm{~m}$ \\
Diámetro del contenedor $=2 \mathrm{~m}$ \\
Altura del contenedor $=1,22 \mathrm{~m}$ \\
\hline Capacidad de secado $=$ hasta $750 \mathrm{Kg}$ \\
\hline Material de construcción Acero inoxidable AISI 304, acero estructural A36 \\
\hline Motorreductor trifásico de $3 \mathrm{HP}$, fuente de corriente eléctrica de $220 \mathrm{~V}$ \\
\hline Peso total de la máquina $865,52 \mathrm{Kg}$ \\
\hline Tamiz: Plancha perforada de acero inoxidable AISI 304 \\
\hline
\end{tabular}

\section{Resultados}

Mediante las pruebas realizadas del secado de cacao en la máquina se tiene los siguientes resultados. Después de un proceso de fermentación del cacao que es de dos a tres días el resultado queda con un porcentaje de humedad entre el 55 - $60 \%$, para posteriormente ser llevados a la máquina secadora automática.

Pruebas de secado de cacao con carga. - Para determinar la curva de secado del cacao, se realizó las pruebas correspondientes de humedad en el secador de lecho fluidizado con la muestra de $1 \mathrm{Kg}$. En la Figura 5 se muestra el tipo de secadero utilizado.

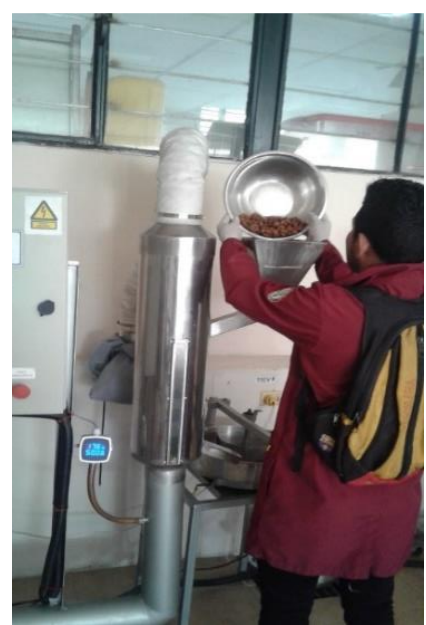

Figura 5. Secador de lecho fluidizado 
Después de 10 minutos que se estabiliza el secador de lecho fluidizado se toma muestras cada 5 minutos para tabular el porcentaje de humedad en el producto y se obtuvieron los siguientes resultados, en la Tabla 7.

Tabla 7. Resultado del porcentaje de humedad del cacao

\begin{tabular}{|l|l|l|}
\hline $\begin{array}{l}\text { Tiempo } \\
\text { (min.) }\end{array}$ & $\begin{array}{l}\text { Humedad } \\
(\%)\end{array}$ & $\begin{array}{l}\text { Temperatura } \\
\left({ }^{\circ} \mathbf{C}\right)\end{array}$ \\
\hline 0 & 9,4 & 43,5 \\
\hline 5 & 9,2 & 43,8 \\
\hline 10 & 9,1 & 44,1 \\
\hline
\end{tabular}

Una vez realizadas las pruebas de secado en la máquina rotativa con una duración de 4 horas, su resultado final se puede observar en la Figura 6.
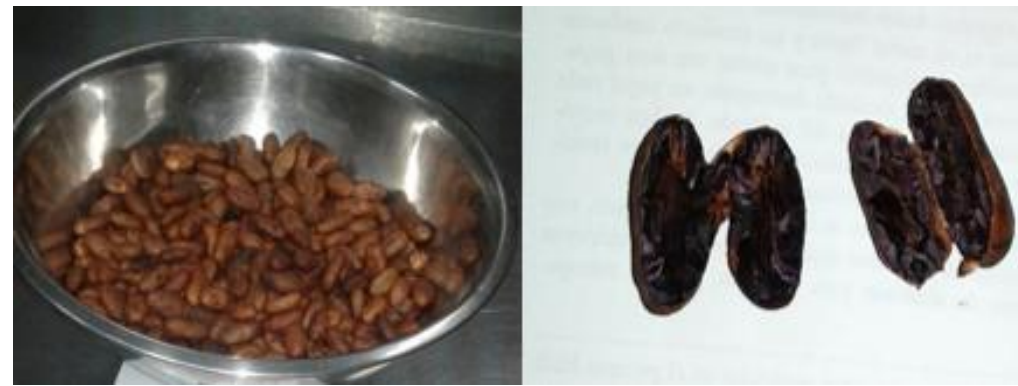

Figura 6. Resultados del cacao seco

El resultado final de porcentaje de humedad como se puede apreciar en la Tabla 7 está en un rango de $9,1-9,4 \%$, con una temperatura promedio de $43,5-44,1^{\circ} \mathrm{C}$, lo que resulta que la máquina secadora automática de cacao para obtener este porcentaje de humedad tiene que estar en el proceso de secado entre 4 a 5 horas.

Para las pruebas de secado se consideró un peso inicial de $200 \mathrm{lb}$ (2 quintales), con un porcentaje de humedad entre $55-60 \%$, obtenido un producto con un $9 \%$ de humedad final (Tabla 8 ).

Tabla 8. Resultado inicial y final de humedad del cacao

\begin{tabular}{|l|l|l|l|}
\hline Peso inicial (lb) & Peso final (lb) & Humedad inicial (\%) & Humedad final (\%) \\
\hline 200 & 100 & $55-60$ & 9 \\
\hline
\end{tabular}

Para visualizar el comportamiento de la curva de secado del cacao humedad, temperatura en función del tiempo, se realiza con granos de cacao que no estén secos totalmente, en este caso con un grado de fermentación y humedad del $35,6 \%$ y se lo secará hasta conseguir un porcentaje adecuado entre 9 al $10 \%$ para lo cual se toman datos cada 10 minutos; los resultados se muestran en la Figura 7.

Pruebas del secado manual.- La siguiente prueba se realizó a una temperatura de $25^{\circ} \mathrm{C}$ en el transcurso de 4 horas diarias (entre las 9:00 y las 16:00 horas) durante 7 días.

En la Figura 8 se observa cómo la humedad de los granos de cacao disminuye desde un $73,6 \%$ hasta un $11,4 \%$ en un tiempo de 7 días, además el peso disminuye desde las $200 \mathrm{lb}$ que es el peso de la muestra hasta 104,5 lb en un tiempo de 7 días, en la Tabla 9, se muestran las condiciones del clima y suelo.

Recordar que para el proceso de secado manual se depende fundamentalmente de la temperatura, radiación solar, humedad relativa del lugar que se haya realizado la prueba. 


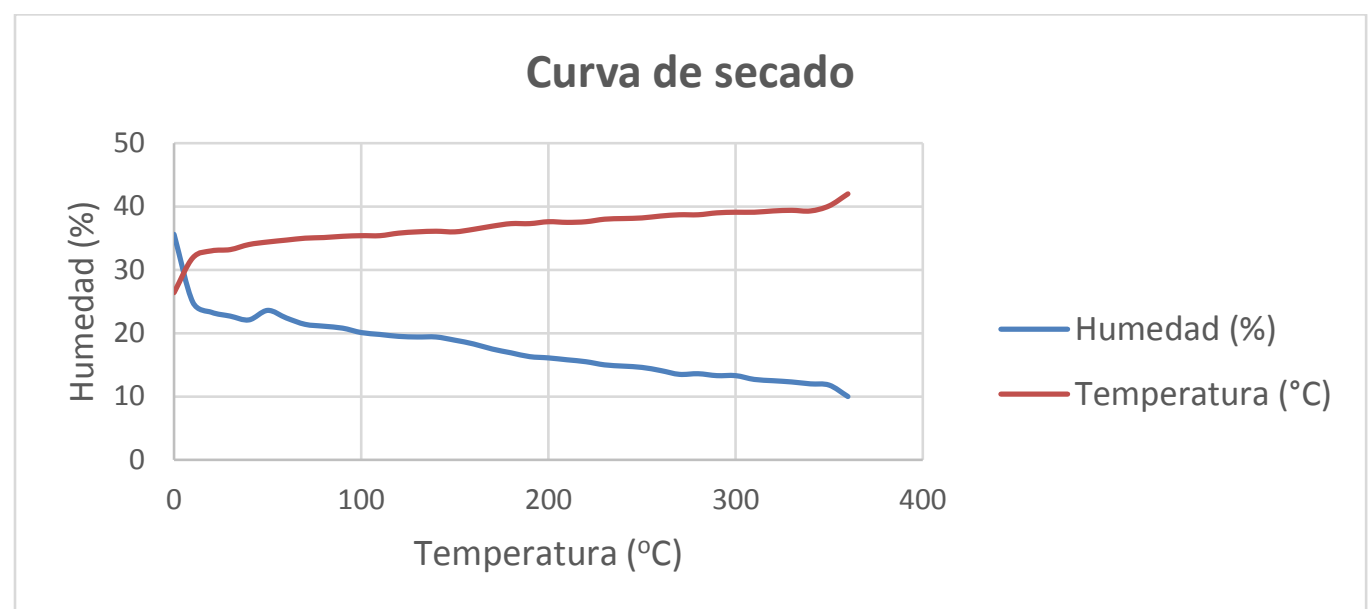

Figura 7. Curva de secado del cacao

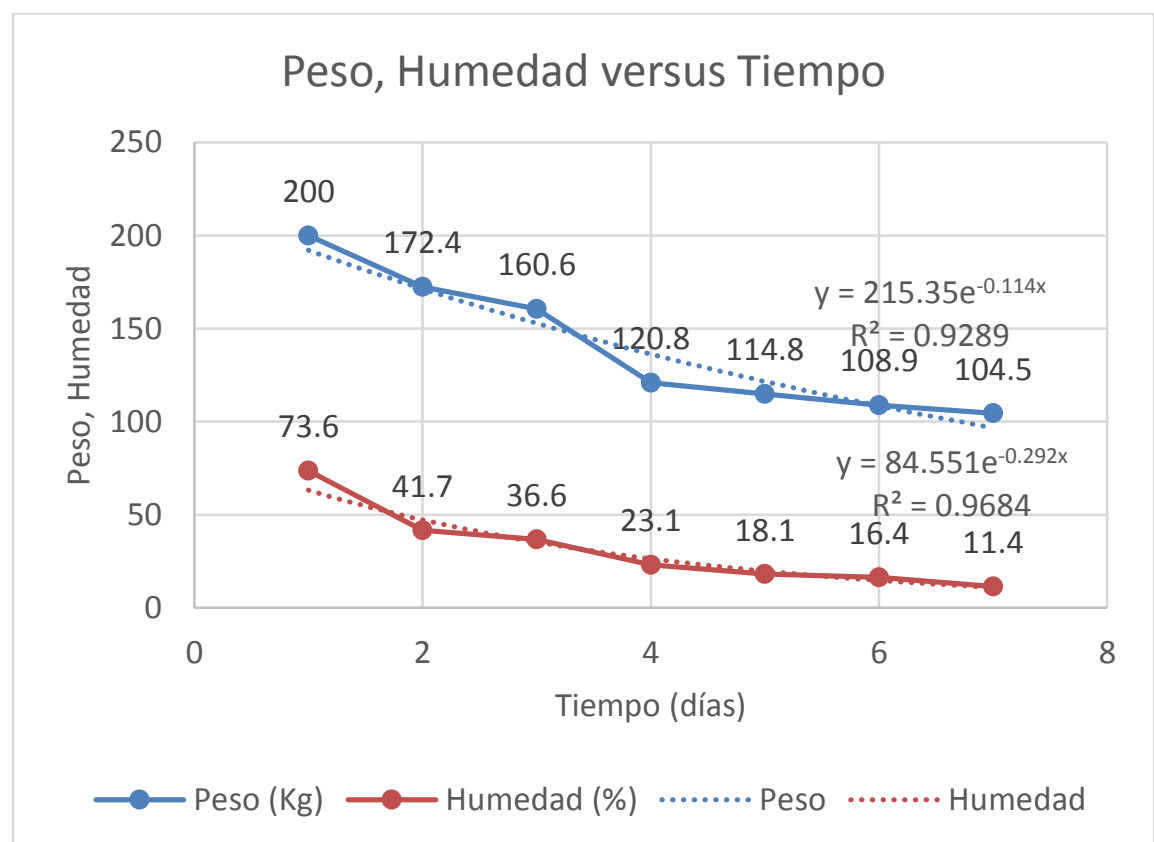

Figura 8. Curva Peso, Humedad versus Tiempo

Tabla 9. Condiciones de clima y suelo

\begin{tabular}{|l|l|l|}
\hline Condiciones de & Clima y Suelo para la Producción de Cacao \\
\hline Condición & Característica & Valor \\
\hline \multirow{4}{*}{ Temperatura } & Rango Anual & 17 a $29^{\circ} \mathrm{C}$ \\
\cline { 2 - 3 } & Límite Inferior & Mayor a $15^{\circ} \mathrm{C}$ \\
\cline { 2 - 3 } & Límite Superior & Menor de $30^{\circ} \mathrm{C}$ \\
\cline { 2 - 3 } & Oscilación día - noche & Menor a $9{ }^{\circ} \mathrm{C}$ \\
\hline \multirow{3}{*}{$\begin{array}{l}\text { Humedad } \\
\text { Relativa }\end{array}$} & Rango Anual & $70-80 \%$ \\
\cline { 2 - 3 } & Límite Superior (evita plagas / & $80 \%$ \\
& enfermedades) & \\
\hline
\end{tabular}

Fuente: (ESPINOZA MARTÍNEZ \& RUIZ BUITRÓN, 2015)

Al verificar en la secadora automática se obtiene una humedad del $9 \%$ en 5 horas, es decir se han ganado 6 días de trabajo, como se muestran en la Tabla 10. Además, serían 6 días de trabajo tomando en cuenta que todos los días hayan tenido un clima adecuado. 
Tabla 10. Comparación de Resultados

\begin{tabular}{|l|l|l|l|}
\hline $\begin{array}{l}\text { TIPO DE } \\
\text { SECADO }\end{array}$ & $\begin{array}{l}\text { DÍAS DE } \\
\text { SECADO }\end{array}$ & Humedad \% & $\begin{array}{l}\text { Peso Final } \\
\text { (Ib) }\end{array}$ \\
\hline $\begin{array}{l}\text { SECADO } \\
\text { MANUAL }\end{array}$ & 7 & 11,4 & 104,5 \\
\hline $\begin{array}{l}\text { SECADO } \\
\text { AUTOMÁTICO }\end{array}$ & 1 (4-5 Horas) & 9 & 43,8 \\
\hline
\end{tabular}

Se verifica que la mejor opción a tomar es secar los granos de cacao mediante el proceso artificial para obtener una humedad de $9 \%$ en 5 horas de trabajo ganando eficacia en la productividad.

\section{Comparación de Estudios}

La propuesta de diseñar y construir el prototipo de la unidad secadora debe ser analizada desde el punto de vista técnico y operacional. Con la ayuda del software se realizó un estudio técnico en el que se ve el tamaño ideal de la secadora de granos y se debe realizar un estudio de consumo energético y consumo de combustible de la unidad para secar $441 \mathrm{lb}$. (GONZÁLEZ, TORRES, 2016). En la Tabla 11 se pueden ver los elementos, potencia y costos de operación de la máquuina.

Tabla 11. Potencia de los equipos, costo de operación

\begin{tabular}{|l|l|l|}
\hline ELEMENTO & CARACTERÍSTICAS & EQUIVALENCIA (Kw) \\
\hline Motor & $1 / 2 \mathrm{Hp}$ & 0,373 \\
\hline Ventilador & $1 \mathrm{Hp}$ & 0,746 \\
\hline Potencia Instalada & $11 / 2 \mathrm{Hp}$ & 1,119 \\
\hline
\end{tabular}

\begin{tabular}{|l|ll|l|}
\hline $\begin{array}{l}\text { POTENCIA } \\
\text { INSTALADA }\end{array}$ & $\begin{array}{l}\text { HORAS } \\
\text { OPERACIÓN }\end{array}$ & DE & PRECIO (Kwh) \\
\hline 1,119 & 11,38 & & 0,1 \\
\hline $\begin{array}{l}\text { TOTAL COSTO POR HORA } \\
\text { OPERACIÓN }\end{array}$ & DE & 1,273422 \\
\hline
\end{tabular}

Fuente: (GONZÁLEZ, TORRES, 2016)

El quemador utilizará 2,14 $\mathrm{Kg}$ de GLP por hora trabajada, dando un total de 24,32 $\mathrm{Kg}$ de GLP por el tiempo total de secado. El costo del GLP es de $\$ 2.00$ (15 Kg), esto da un total de $\$ 3,25$ por operación de secado.

Consumo energético y consumo de combustible de la unidad de investigación para secar $441 \mathrm{lb}$, en la Tabla 12 se muestran los valores establecidos en el prototipo.

Tabla 12. Potencia de los equipos, costo de operación. ACTUALES

\begin{tabular}{|l|l|l|}
\hline ELEMENTO & CARACTERÍSTICAS & EQUIVALENCIA (Kw) \\
\hline Motor & $3 \mathrm{Hp}$ & 2,238 \\
\hline Ventilador & $3 / 4 \mathrm{Hp}$ & 0,5595 \\
\hline Potencia Instalada & $11 / 2 \mathrm{Hp}$ & 2,7975 \\
\hline
\end{tabular}

\begin{tabular}{|l|ll|l|}
\hline $\begin{array}{l}\text { POTENCIA } \\
\text { INSTALADA }\end{array}$ & $\begin{array}{l}\text { HORAS } \\
\text { OPERACIÓN }\end{array}$ & DE & PRECIO (Kwh) \\
\hline 2.7975 & 5 & & 0,08 \\
\hline $\begin{array}{l}\text { TOTAL COSTO POR HORA } \\
\text { OPERACIÓN }\end{array}$ & DE & 1,119 \\
\hline
\end{tabular}


El quemador utilizará $1,69 \mathrm{Kg}$ de GLP por hora trabajada, dando un total de $8,43 \mathrm{Kg}$ de GLP por el tiempo total de secado. El costo del GLP es de $\$ 2.00(15 \mathrm{Kg})$, esto da un total de $\$ 1,12$ por operación de secado, reduciendo en un $65 \%$ en costos de operación y ganando tiempo de eficacia para la producción de cacao en comparación al trabajo (GONZÁLEZ, TORRES, 2016).

\section{Discusión}

Se ha construido una máquina secadora para cacao, la cual cumple con los requerimientos establecidos por el cliente final, en la metodología de diseño se ha tomado en cuenta el diseño mecánico y energético, una vez construido el equipo se procedió a las pruebas siguiendo el protocolo de pruebas correspondiente obteniendo resultados satisfactorios. En las pruebas de secado el producto obtuvo un porcentaje de humedad del $9 \%$, valor que está dentro del rango requerido.

\section{Conclusiones y recomendaciones}

Mediante las pruebas realizadas la secadora tipo rotatoria cumple con los requerimientos del usuario mejorando la eficiencia y producción en el sector campesino.

El resultado obtenido en la secadora después del proceso de secado, el cacao quedó con un porcentaje de humedad del $9 \%$ alcanzando una temperatura entre 43 a $44^{\circ} \mathrm{C}$; este dato se verificó en el laboratorio de procesos industriales, cumpliendo de esta manera con los estándares de calidad del producto.

Aplicando la metodología de diseño se elaboró la matriz QFD (Casa de la Calidad), cumpliendo la voz del usuario y requerimientos técnicos del ingeniero mediante las pruebas de funcionamiento.

Para realizar el diseño energético se consideraron las propiedades del aire en la provincia de Santo Domingo de los Tsáchilas lugar donde se va a instalar la máquina y con el uso del software Engineering Equation Solver (EES) se determinan las propiedades del aire al inicio y final de secado para su posterior cálculo.

La máquina cumplió con las normas sanitarias y estándares de calidad, en lo que se refiere alimentos en nuestro caso por ser un producto alimenticio su construcción se realizó en acero inoxidable AISI 304 la parte que está en contacto con el producto.

El costo total de la máquina es de 10217 USD incluyendo los costos directos e indirectos, para la viabilidad del proyecto se determinó la tasa interna de retorno (TIR) la cual fue del 76,76\% que garantiza la construcción de la máquina al usuario.

La comparación realizada entre la máquina artesanal con la automática resulto más eficiente la automática evaluando el tiempo de secado, mano de obra y volumen de producción.

La secadora automática para cacao cuenta con un variador de frecuencia el cual se puede variar a la velocidad que se requiere para realizar el secado lo que garantiza mantener las propiedades del producto y rapidez de secado, esto no sucede en una máquina artesanal.

\section{Bibliografía}

ASME. Rules for construction of pressure vessels. New York : s.n., 2013.

Budynas, Richard \& Nisbett, Keith. Diseño en ingeniería mecánica de Shigley.10 ed. México : McGraw-Hill, 2014. 0073398209.

ECUADOR, PRO. Análisis del sector cacao y elaborados. [En línea] 2013. [Consultado el: 06 de junio de 2016]. Disponible en: http://www.proecuador.gob.ec/wpcontent/uploads/2013/08/PROEC_AS2013_CACAO.pdf.

ECUADOR, PRO. Perfil sectorial de cacao y elaborados. [En línea] 2015. [Consultado el: 15 de junio de 2016]. Disponible en http://www.fao.org/3/a-au995s.pdf. 
Espinosa, Cristina \& Ruiz, Valeria. Diseño y Construcción de una máquina secadora de cacao para aplicación agroindustrial en la provincia de Santo Domingo de los Tsáchilas. [En línea] (Tesis) Universidad de las Fuerzas Armadas, Departamento de Ciencias de la Energía y Mecánica, Escuela de Ingeniería Mecatrónica, Sangolquí, Ecuador. 2015. [Consultado el: 20 de junio de 2016]. Disponible en: http://repositorio.espe.edu.ec/xmlui/handle/21000/10323.

Garcia A, S. A. (2017). Moisture acidity and temperature evolution during cacao drying. 79(79).

GONZALÉS VÁZQUES, Á. (2012). Modelo de empresa asociativa acopiadora de cacao fino de aroma para los productores del Cantón Quinsaloma. Recuperado el 15 de junio de 2016, de http://dspace.ups.edu.ec/bitstream/123456789/2748/6/UPSGT000274.pdf

HAUSER, G., CURIEL, G., BELLIN, W., CNOSSEN, H., HOFMANN, J., KASTELEIN, J., TIMPERLEY, A. (2004). Criterios para el diseño higiénico de equipos. Recuperado el 26 de julio de 2016, de https://www.ehedg.org/fileadmin/guidelines /DOC_08_SP_2004.pdf

Holman, J.P. Transferencia de calor. 10a ed. México: McGraw-Hill, 1999. 968-26-0649-7.

JÁCOME BAZURTO, M. (2010). Incidencia de la aplicación de tecnología de secado en el mejoramiento del valor agregado del cacao (Theobroma cacao) variedad CCN-51. Recuperado el 20 de junio de 2016, de http://www.censalud.ues.edu.sv/CDOCDeployment/documentos/INCIDENCIA_DE_LA_APLICACI\%C3\%93N_DE_

Jimbo, César \& Arboleda, Freddy. Diseño mécanico y construcción de un secador de cacao (Theobroma caao L.) al vacío para medianos y grandes productores en la Provincia de Manabí. [En línea] (Tesis) Escuela Superior Politecnica del Litoral, Facultad de Ingeniería en Mecánica y Ciencias de la Producción, Guayaquil, Ecuador. 2016. [Consultado el: 20 de junio de 2016]. Disponible en: http://www.dspace.espol.edu.ec/xmlui/bitstream/handle/123456789/34444/DCD883 04.pdf? sequence $=-1$ \&isAllowed $=\mathrm{y}$.

Jordán, Joann. Analizar y validar un programa de rehabilitación en la poscosecha del cacao CCN51, en la Finca Rami, en la Provincia de los Ríos. [En línea] (Tesis) Escuela Superior Politécnica del Litoral, Facultad de Ingeniería en Mecánica y Ciencias de la Producción, Guayaquil, Ecuador. 2013. [Consultado el: 23 de junio de 2016]. Disponible en: https://www.dspace.espol.edu.ec/retrieve/89728/D79835.pdf.

Lannes S, Caetano da Silva. Processamento de achocolatado de cupuaçu por spraydryer. Rev. Bras. Cienc. Farm. [online]. 2003, vol.39, n.1, pp.115-123. Available from: http://dx.doi.org/10.1590/S1516-93322003000100012.

Macmanus, N; Ogunlowo, A y Olukunle, O. Cocoa Bean (Theobroma cacao L.) Drying Kinetics. Chilean J. Agric. Res. [online]. 2010, vol.70, n.4, pp.633-639. Disponible en: http://dx.doi.org/10.4067/S0718-58392010000400014.

NORTON, R. L. (2009). Diseño de maquinaria (4a ed ed.). México: McGraww-Hill.

Perez, Mario; et al. Introducción a los sistemas de control y modelo matemático para sistemas lineales invariantes en el tiempo. [En línea] (Tesis) Universidad Nacional de San Juan, Facultad de Ingeniería, Escuela de Ingeniería Electrónica y Automática. 2007. [Consultado el: 22 de julio de 2016]. Disponible en: http://dea.unsj.edu.ar/control1/apuntes/unidad1y2.pdf.

PLAZA ESPINOZA, X., \& YANGUE ZAMBRANO, W. (2012). Diseno e implementación de una secadora híbrida para el control y monitoreo del proceso de secado del cacao. Recuperado el 15 de julio de 2016, de http://dspace.ups.edu.ec/bitstream/123456789/2748/6/UPS-GT000274.pdf

RIBA ROMEVA, C. (2002). Diseño concurrente (1a ed ed.). Barcelona.

Ruiz, Arturo \& Rojas, Falcó. Despliegue de la función calidad (QFD). [En línea] 2009. [Consultado el: 20 de agosto de 2016]. Disponible en: http://web.cortland.edu/matresearch/QFD.pdf. 
Ruiz, Jorge. Secadoras de cacao SIRCA (Ecuador). [En línea] 2015. [Consultado el: 4 de julio de 2016]. Disponible en: http://www.canacacao.org/contenido.item.409 /secadoras-de-cacao-sirca-ecuador.html.

Teixeira-Da Silva, J. y Malpica, F. Desarrollo de un modelo matemático para dimensionar un deshidratador solar directo de cacao. Ingeniería Mecánica [online]. 2016, vol.19, n.1, pp. 30-39 Disponible en: $<$ http://scielo.sld.cu/scielo.php?script=sci_arttext\&pid=S181559442016000100005\&lng=es\&nrm=iso >. ISSN 1815-5944.

Tirida, Lenin \& Castro, Diego. Diseño y Construcción de una máquina despulpadora de frutas. [En línea] (Tesis) ESPOCH, Facultad de Mecánica, Escuela de Ingeniería Mecánica, Riobamba, Ecuador. 2014. [Consultado el: 10 de agosto de 2016]. Disponible en: http://dspace.espoch.edu.ec/bitstream/123456789/3715/1 /15T00586.pdf.

Vissotto, F. et al. Morphological characterization with image analysis of cocoa beverage powder agglomerated with steam. Food Sci. Technol (Campinas)[online]. 2014, vol.34, n.4, pp.649-656. Available from: http://dx.doi.org/10.1590/1678-457X.6246.

ZABALA, G. (2012). Termodinámica II (10a ed ed.). Riobamba, Ecuador. 